\title{
Effects of Familial Mediterranean Fever on Cardiac Functions in Adults: A Cross-Sectional Study Based on Speckle Tracking Echocardiography
}

\author{
Hilal ERKEN PAMUKCU ${ }^{1}\left(\mathbb{D}\right.$, Mehmet DOĞAN $^{2}$, Cem ÖZİŞLER ${ }^{3}$, Hamza SUNMAN ${ }^{1}$, \\ Melih PAMUKCU ${ }^{4}$ (D) Lale DİNÇ ASARCIKLI ${ }^{5}$ (D) \\ ${ }^{1}$ Department of Cardiology, Dışkapı Yıldırım Beyazıt Training and Research Hospital, Ankara, Turkey \\ ${ }^{2}$ Department of Cardiology, Istanbul Medipol University, İstanbul, Turkey \\ ${ }^{3}$ Department of Rheumatology, Dışkapı Yıldırım Beyazıt Training and Research Hospital, Ankara, Turkey \\ ${ }^{4}$ Department of Rheumatology, Malatya Training and Research Hospital, Malatya, Turkey \\ ${ }^{5}$ Department of Cardiology, Dr. Siyami Ersek Thoracic and Cardiovascular Surgery Training and Research Hospital, Istanbul, Turkey
}

\begin{abstract}
Objectives: This study aims to evaluate the right ventricular (RV) and left ventricular (LV) systolic and diastolic functions with speckle tracking echocardiography in addition to routine echocardiographic measurements in adult familial Mediterranean fever (FMF) patients in order to detect cardiac functions.

Patients and methods: Sixty FMF patients ( 23 males, 37 females; median age 35 years; interquartile range, 26 to 38 years) and 20 healthy subjects (10 males, 10 females; median age 31 years; interquartile range, 25 to 35 years) were included in the study. The diagnosis was established according to the Tel-Hashomer criteria. All patients were using regular colchicine and they were in the attack-free period. Laboratory examinations included complete blood count, creatinine, and inflammatory markers. In addition to routine echocardiographic examination, RV and LV global longitudinal strains were measured and compared.

Results: Erythrocyte sedimentation rate and C-reactive protein values were higher in FMF group. LV global longitudinal strain was similar among the groups. FMF patients had slightly lower early diastolic trans-mitral flow (E) values than controls. As similar as the mitral E flow, tricuspid E flow was slightly lower in FMF groups than controls. RV ejection fraction was similar and in normal ranges among the groups. RV global longitudinal strain was lower in FMF group than controls. RV Myocardial Performance Index (or Tei index) was higher in FMF group.

Conclusion: The present study indicates low values of mean RV global longitudinal strain and higher Tei index in FMF patients. These results suggest that FMF may cause subclinical RV deterioration.

Keywords: Familial Mediterranean fever, speckle tracking, strain echocardiography, Tei index.
\end{abstract}

Familial Mediterranean fever (FMF) is an autosomal recessive hereditary autoinflammatory disorder which is characterized by recurrent fever attacks and serosal inflammation. ${ }^{1}$ FMF was originally described among individuals of the Mediterranean basin. ${ }^{2}$ However, the disease was reported in other ethnicities at a lower prevalence. . $^{3-5}$ Inflammation in pleura and pericardium or joints can also occur. Kees et al. ${ }^{6}$ reported that the coexistence of pericarditis is $0.7 \%$. FMF can cause long-term complications such as secondary amyloidosis, which decrease with the regular use of colchicine. Moreover, some studies claim that FMF can cause atherosclerosis. ${ }^{7}$

Received: May 07, 2018 Accepted: September 11, 2018 Published online: November 30, 2018

Correspondence: Hilal Erken Pamukcu, MD. Dışkapı Yıldırım Beyazıt Eğitim ve Araştırma Hastanesi Kardiyoloji Kliniği, 06110 Dışkapı, Ankara, Turkey. Tel: +90 532 - 7813714 e-mail: hilalerkenn@gmail.com 
Endothelial dysfunction has been demonstrated with ultrasound in FMF patients. ${ }^{8}$ There are some studies that claim FMF is in association with diastolic dysfunction. ${ }^{9,10}$ However, myocardial function has been investigated in FMF patients and diverse results were obtained from different studies. In a study, FMF was found to be in relation with reduced left ventricular (LV) strain in children, ${ }^{11}$ but there is lack of research that shows the relationship between FMF and systolic function in adults.

In this study, we aimed to evaluate the right ventricular (RV) and LV systolic and diastolic functions with speckle tracking echocardiography (STE) in addition to routine echocardiographic measurements in adult FMF patients in order to detect cardiac functions.

\section{PATIENTS AND METHODS}

This single-center, cross-sectional study was conducted in the central region of Turkey, at University of Health Sciences, Dışkapı Yıldırım Beyazit Training and Research Hospital between March 2018 and April 2018. Sixty FMF patients (23 males, 37 females; median age 35 years; interquartile range [IQR], 26 to 38 years) and 20 healthy subjects (10 males, 10 females; median age 31 years; IQR, 25 to 35 years) were included. Control group consisted of healthy individuals who were free of chronic diseases, diabetes mellitus, hypertension, and alcohol abuse, etc. They were selected from the hospital staff who are examined periodically. The study protocol was approved by the University of Health Sciences, Diskapi Yildirim Beyazit Training and Research Hospital Ethics Committee (19/3/2018-47/26). A written informed consent was obtained from each participant. The study was conducted in accordance with the principles of the Declaration of Helsinki.

Familial Mediterranean fever diagnosis was established according to the Tel-Hashomer criteria. ${ }^{12}$ All FMF patients were under regular colchicine treatment at a dose of $1-1.5 \mathrm{mg} /$ day and all of them were in the attack-free period during echocardiographic evaluation. None of the FMF patients had experienced pericarditis before. The clinical data including age, sex, age at onset and dosage of colchicine were obtained. Laboratory examinations included complete blood count, erythrocyte sedimentation rate (ESR), C-reactive protein (CRP), and fibrinogen.

Patients who experienced less than three FMF attacks per year were included. Patients on biologic drugs were excluded. None of the patients had subclinical inflammation at one-year follow-up. ESR, CRP, and fibrinogen levels were within normal ranges during attack-free phase. According to genetic mutations, 20 patients were homozygous (MEFV $694 \mathrm{~V}$ ), 11 patients were heterozygous, and 29 patients were compound heterozygotes.

Patients with other inflammatory diseases, moderate or severe heart valve abnormalities, proteinuria, hypertension, renal failure, diabetes mellitus, cardiomyopathy, coronary artery disease, chronic obstructive pulmonary disease, or those who were smokers, chronic alcohol users or pregnant were excluded. Amyloidosis was excluded from the absence of proteinuria in complete urine analysis and in 24 hours urine test. Transthoracic echocardiographic examination was performed using the Philips Epic 5 (Philips Healthcare, Andover, Massachusetts, USA) instrument with a 1-5 MHz transducer. Standard parasternal long and short-axis views, and apical two and fourchamber views were obtained for all patients. $\mathrm{LV}$ and left atrial diameter were measured from the M-mode images in parasternal long axis view. ${ }^{13}$ Peak tricuspid regurgitant velocities were recorded by the continuous wave Doppler technique and a modified Bernoulli equation was used to estimate pulmonary artery systolic pressure (PASP). The modified Simpson's method was used for calculating the LV ejection fraction (LVEF) using the apical four-chamber views. ${ }^{13}$ From the apical window, a $2 \mathrm{~mm}$ pulsed Doppler sample volume was placed at the mitral valve tip and mitral flow velocities of three cardiac cycles were recorded by obtaining peak velocities of the early diastolic trans-mitral flow (E) and late diastolic trans-mitral flow (A). Additionally, early diastolic lateral mitral annulus velocity $\left(\mathrm{E}_{\text {lateral }}^{\prime}\right)$, velocity during atrial contraction $\left(\mathrm{A}_{\text {lateral }}^{\prime}\right)$ and lateral systolic $(S)$ myocardial velocity were measured by tissue Doppler imaging (TDI) using the pulsed wave Doppler. ${ }^{14}$

Right ventricle free wall TDI allows quantitative assessment of both RV systolic and diastolic 
function. Myocardial systolic velocity (S) correlates with the RV systolic function. From the apical window, a $2 \mathrm{~mm}$ pulsed Doppler sample volume was obtained from lateral tricuspid annulus and early diastolic annulus velocity $\left(\mathrm{E}_{\text {tricuspid }}\right.$ ) during atrial contraction $\left(\mathrm{A}_{\text {tricuspid }}^{\prime}\right)$ and lateral systolic myocardial velocity was measured by TDI using the pulsed wave Doppler. ${ }^{14}$

The Tei index (also known as myocardial performance index), described by Tei et al., ${ }^{15}$ is a Doppler-derived time interval index that combines both systolic and diastolic cardiac performance. The Tei index was calculated by the following formula: (isovolumic contraction time + isovolumic relaxation time)/ejection time. RV Tei index was measured by using pulsed tissue Doppler imaging of the RV free wall. ${ }^{14}$

Two-dimensional (2D) global longitudinal strain (GLS) echocardiography images were obtained from standard apical four-chamber, three-chamber, and two-chamber views of the LV from the apex. Three stable cardiac cycles were stored for each view and all data were collected and studied on the echocardiography machine's own program (QLAB 10.1, Philips Healthcare, Andover, Massachusetts, USA) as to offline analysis. Frame rates used for GLS analysis were $40-80$ frames/s. ${ }^{16}$ Conventional $2 \mathrm{D}$ grayscale echocardiographic images were used by the system and activity of the speckles was tracked throughout the myocardial tissue. The regions of interest (ROIs) were manually outlined by marking the endocardial borders at the mitral annulus level as well as at the apex of each digital loop; the epicardial surface was automatically generated by the software system. After any required manual adjustment, the ROIs were divided into six segments. Each segment was then scored automatically by the software according to the image quality. The peak systolic strain values in an 18 -segment LV model were used. ${ }^{16}$ The results for all three planes were then combined in a single bulls-eye summary that provided the GLS. Measurements were repeated at least three times and the average of these measurements was determined. GLS rate was measured by the same technique.

Right ventricular strain was measured by $2 \mathrm{D}$ STE on the RV-focused apical four-chamber view using QLAB package program (QLAB 10.1; Philips Healthcare, Andover, Massachusetts, USA). After any required manual adjustment, the ROIs were divided into six segments. The software automatically divided the RV free wall and the interventricular septum into three segments each (basal, mid, and apical), resulting in a six-segment model.

\section{Statistical analysis}

Statistical analyses were performed using the IBM SPSS version 20.0 software (IBM Corp., Armonk, NY, USA). Kolmogorov-Smirnov test was used to determine whether the data distribution was normal or not. Descriptive statistics were expressed as median (interquartile ranges) for

Table 1. Demographic and biochemical features of FMF and control groups

\begin{tabular}{|c|c|c|c|c|c|c|c|}
\hline & \multicolumn{3}{|c|}{ FMF patients $(n=60)$} & \multicolumn{3}{|c|}{ Controls $(n=20)$} & \multirow[b]{2}{*}{$p$} \\
\hline & $\%$ & Median & IQR & $\%$ & Median & IQR & \\
\hline Age (year) & & 35 & $26-38$ & & 31 & $25-35$ & NS \\
\hline $\begin{array}{l}\text { Gender } \\
\text { Female }\end{array}$ & 61.7 & & & 50 & & & NS \\
\hline Hemoglobin (g/dL) & & 14 & $12.8-15.2$ & & 13.3 & $12.2-14.8$ & 0.05 \\
\hline WBC $\left(10^{9} / \mathrm{L}\right)$ & & 6.8 & $5.8-8.1$ & & 6.7 & $5.9-7.4$ & NS \\
\hline Neutrophils $\left(10^{9} / \mathrm{L}\right)$ & & 4.2 & $31-4.8$ & & 4.2 & $4-5.2$ & NS \\
\hline Lymphocyte (109/L) & & 2.1 & $1.76-2.8$ & & 1.6 & $1.4-1.8$ & $<0.001^{*}$ \\
\hline Creatinine (mg/dL) & & 0.89 & $0.7-0.9$ & & 0.9 & $0.8-0.9$ & NS \\
\hline $\operatorname{ESR}(\mathrm{mm} / \mathrm{h})$ & & 10 & $7-20$ & & 6 & $4-8.5$ & $<0.002^{*}$ \\
\hline $\mathrm{CRP}(\mathrm{mg} / \mathrm{L})$ & & 5.7 & $2.9-10$ & & 5 & $4-6$ & NS \\
\hline Fibrinogen (mg/dL) & & 311 & $250-354$ & & 311 & $250-354$ & $<0.02^{*}$ \\
\hline
\end{tabular}

FMF: Familial Mediterranean fever; IQR: Interquartile range; NS: Not significant; WBC: White blood cells; ESR: Erythrocyte sedimentation rate; CRP: C-reactive protein; * Statistically significant. 
non-normally distributed data. Categorical and ordinal variables were given as percentages. Mann-Whitney $\mathrm{U}$ test was performed to analyze the significance of the differences of medians, and the differences were studied using post hoc tests. A $p$ value $<0.05$ was considered statistically significant.

\section{RESULTS}

Characteristics of FMF and control groups are given in Table 1. Mean disease duration was
$15.1 \pm 9.3$ years and mean colchicine dosage was $1.42 \pm 0.2 \mathrm{mg} /$ day. There was no statistically significant difference between groups in terms of age, sex, white blood cells and creatinine levels. However, hemoglobin 14 (IQR: 12.8-15.2; $\mathrm{p}=0.05)$ vs. 13.3 (IQR: 12.2-14.8; $\mathrm{p}=0.05$ ), lymphocyte 2.1 (IQR: 1.76-2.8; $\mathrm{p}<0.001$ ) vs. 1.6 (IQR: 1.4-1.8; p<0.001), ESR 10 (IQR: 7-20; $\mathrm{p}<0.002 *)$ vs. 6 (IQR: 4-8.5; $\mathrm{p}<0.002^{*}$ ) and fibrinogen 311 (IQR: 250-354; $\mathrm{p}<0.02$ ) vs. 311 (IQR: 250-354; $\mathrm{p}<0.02$ ) levels were higher in the FMF group than controls (Table 1).

Table 2. Echocardiographic findings of FMF and control groups

\begin{tabular}{|c|c|c|c|c|c|}
\hline & \multicolumn{2}{|c|}{ FMF group $(n=60)$} & \multicolumn{2}{|c|}{ Control group $(n=20)$} & \multirow[b]{2}{*}{$\mathrm{p}$} \\
\hline & Median & IQR & Median & IQR & \\
\hline \multicolumn{6}{|l|}{ Groups } \\
\hline LVEF (\%) & 60 & $58.7-62$ & 62 & $60-63$ & $0.003^{*}$ \\
\hline LVEdD $(\mathrm{cm})$ & 4.2 & $3.8-4.4$ & 4.1 & $3.9-4.1$ & NS \\
\hline $\operatorname{LVEsD}(\mathrm{cm})$ & 2.9 & $2.7-3$ & 2.9 & $2.7-3$ & NS \\
\hline $\mathrm{LA}(\mathrm{cm})$ & 2.9 & 2.8-3.2 & 2.9 & $2.8-3.2$ & NS \\
\hline \multicolumn{6}{|l|}{ Mitral inflow } \\
\hline $\mathrm{E}(\mathrm{m} / \mathrm{s})$ & 0.7 & $0.6-0.8$ & 0.8 & $0.7-0.9$ & $0.02^{*}$ \\
\hline $\mathrm{A}(\mathrm{m} / \mathrm{s})$ & 0.6 & $0.5-0.7$ & 0.6 & $0.5-0.7$ & NS \\
\hline Deceleration time (ms) & 175 & $164.2-185$ & 177.5 & $165-185.5$ & NS \\
\hline IVRT (ms) & 68 & $65-70.5$ & 65 & $64.2-70$ & NS \\
\hline \multicolumn{6}{|l|}{ Mitral lateral annulus } \\
\hline E'm peak velocity $(\mathrm{cm} / \mathrm{s})$ & 12.9 & $9.3-16$ & 12.1 & $11.7-13.2$ & NS \\
\hline A'm peak velocity $(\mathrm{cm} / \mathrm{s})$ & 9.2 & $8-12$ & 9.5 & 7.8-12.2 & NS \\
\hline $\mathrm{S}$ m peak velocity $(\mathrm{cm} / \mathrm{s})(>6)$ & 9 & $7-10$ & 10 & $9-10.5$ & $0.03^{*}$ \\
\hline \multicolumn{6}{|l|}{ Tricuspid inflow } \\
\hline Tricuspid E (m/s) & 0.5 & $0.5-0.6$ & 0.5 & $0.5-0.6$ & $0.04^{*}$ \\
\hline Tricuspid A (m/s) & 0.4 & $0.3-0.6$ & 0.4 & $0.3-0.6$ & NS \\
\hline \multicolumn{6}{|l|}{ Tricuspid annulus } \\
\hline E’t peak velocity $(\mathrm{cm} / \mathrm{s})$ & 11.5 & $8-14$ & 10 & $8-14$ & NS \\
\hline A't peak velocity $(\mathrm{cm} / \mathrm{s})$ & 14 & $10.3-16.1$ & 14 & $11-15.3$ & NS \\
\hline $\mathrm{S}$ t peak velocity $(\mathrm{cm} / \mathrm{s})$ & 12.5 & $11-13.2$ & 11.4 & $10.9-14.2$ & NS \\
\hline TAPSE $(\mathrm{cm})$ & 2.4 & $2.3-2.5$ & 2.5 & $2.2-2.6$ & NS \\
\hline PASP $(\mathrm{mmHg})<25$ & 25 & $20-25.2$ & 22 & $20-25$ & NS \\
\hline RVEF (\%) & 54.9 & $53.2-57.4$ & 55 & $53.8-57.6$ & NS \\
\hline Tei index & 0.32 & $0.3-0.36$ & 0.23 & $0.21-0.25$ & $<0.001^{*}$ \\
\hline LV GLS (\%) & -23.5 & $-23--25$ & -28 & $-27--29$ & $<0.001^{*}$ \\
\hline RV GLS (\%) & -23.9 & $-22.5--25$ & -28.1 & $-26.5--30.4$ & $<0.001^{*}$ \\
\hline \multicolumn{6}{|c|}{$\begin{array}{l}\text { FMF: Familial Mediterranean fever; IQR: Interquartile range; LVEF: Left ventricle ejection fraction; LVEdD: Left ventricle end-diastolic diameter; LVEsD: Left } \\
\text { ventricle end-systolic diameter; LA: Left atrium; E: Early diastolic peak velocity; A: Late diastolic peak velocity; IVRT: Isovolumic relaxation time; E'm: Early } \\
\text { diastolic myocardial peak velocity of mitral lateral annulus; A'm: Late diastolic myocardial peak velocity of mitral lateral annulus; S m: Peak systolic velocity of } \\
\text { mitral lateral annulus; E't: Early diastolic myocardial peak velocity of tricuspid annulus; A't: Late diastolic myocardial peak velocity of tricuspid annulus; } \mathrm{t} \text { : } \\
\text { Peak systolic velocity of tricuspid annulus; TAPSE: Tricuspid annular plane systolic excursion; PASP: Pulmonary artery systolic pressure; RVEF: Right ventricle } \\
\text { ejection fraction; LV: Left ventricle; GLS: Global longitudinal strain; NS: Not significant * Statistically significant. }\end{array}$} \\
\hline
\end{tabular}


Left ventricular ejection fraction was slightly lower in the FMF group (60\% IQR: 58.7-62) than controls (62\% IQR: 60-63; $p=0.003)$ but all values were within normal ranges. LV diameters or left atrium size were not statistically significantly different between groups. Moreover, LV deceleration time, isovolumetric relaxation time, early diastolic myocardial peak velocity of the mitral lateral annulus ( $\mathrm{E}^{\prime} \mathrm{m}$ ), late diastolic myocardial peak velocity of the mitral lateral annulus ( $\left.\mathrm{A}^{\prime} \mathrm{m}\right)$ and lateral systolic $(\mathrm{S})$ myocardial velocity were similar for both groups. Although late diastolic trans-mitral flow (A) was not different among groups, FMF patients had slightly lower early diastolic trans-mitral flow (E) values 0.7 (IQR: 0.5-0.6) vs. 0.8 (IQR: 0.7-0.9), p=0.02, respectively, Table 2 .

Right ventricular ejection fraction was similar for both groups. Similarly, tricuspid annular plane systolic excursion, PASP, or late diastolic trans-tricuspid flow (A) were not statistically significantly different among FMF and control groups. As well as the mitral E flow, early diastolic trans-tricuspid flow was slightly lower in FMF groups than controls (0.5 [IQR: 0.5-0.6] vs. 0.5 [IQR: 0.5-0.6], respectively, $\mathrm{p}=0.04)$. Tei index was higher in the FMF group than controls (0.32 [IQR: 0.3-0.36] vs. 0.23 [IQR: 0.21-0.25], respectively, $\mathrm{p}<0.001)$. 2D GLS and $2 \mathrm{D}$ GLS rate were statistically significantly different among the groups. Mean RV strain was measured as $-23.9 \%$ (IQR: -22.5-[-25]) in FMF group and this value was significantly lower than the control group $(-28.1 \%$ IQR: -26.5-[-30.4], $\mathrm{p}<0.001)$.

\section{DISCUSSION}

In the present study, we have investigated cardiac functions in adult FMF patients with echocardiography. Our results have shown that FMF patients have a higher Tei index and lower RV strain than controls. Moreover, LVEF, early diastolic trans-mitral and trans-tricuspid flows were found to be slightly lower in the FMF group. A majority of the previous studies on FMF have been conducted on the child population, so this study may provide promising information about adult patients.

Diastolic dysfunction in FMF patients was shown in some studies. ${ }^{[9,10,17]}$ In our study, there was no clear finding for diastolic impairment. However, a slight but statistically significant decrease in early diastolic trans-mitral and transtricuspid $\mathrm{E}$ wave may indicate an early sign of diastolic dysfunction. Several mechanisms have been suggested to explain diastolic dysfunction. One of the most accepted theories is systemic inflammation which causes early atherosclerosis. ${ }^{17}$ This was supported by studies that have shown an increased intima-media thickness of carotid arteries and decreased endothelium-dependent flow-mediated dilation of the brachial artery. ${ }^{8}$ According to our results, elevated ESR, lymphocyte and fibrinogen levels are concordant with relative systemic inflammation increase. Other possible causes of diastolic impairment are impaired active LV relaxation or reduced LV compliance, reduced passive LV filling, decrease in ventricular preload and vasculitis. ${ }^{9,18}$

Tei index is an independent marker of LV and $\mathrm{RV}$ dysfunction. This index provides information about systolic and diastolic functions of ventricles. It has shown that various cardiovascular diseases are associated with increased Tei index such as pulmonary hypertension, ${ }^{19,20}$ coronary artery disease, ${ }^{21}$ congestive heart failure, ${ }^{15}$ chronic pulmonary obstructive disease $e^{22}$ and cardiac amyloidosis. ${ }^{23}$ Amyloidosis is one of the most fatal consequences of FMF. Kim et $\mathrm{al}^{23}$ have previously reported that cardiac amyloidosis is associated with increased RV Tei index. ${ }^{24}$ In our study population, RV Tei index of FMF patients was increased. To the best of our knowledge, this is the first report describing the association between FMF and increased RV Tei index in adult population.

The LV systolic and diastolic functions were analyzed using conventional echocardiography techniques (2D, Doppler, and tissue Doppler), and systolic functions were also studied using 2D STE. GLS is an advanced echocardiography technique and can determine the possible systolic dysfunction in the initial phase before the decline of the LVEF and the occurrence of apparent heart failure. GLS can also be used to determine RV systolic function in many diseases. Previously published studies have demonstrated that $\mathrm{RV}$ strain analysis provides important diagnostic and prognostic benefits in pulmonary hypertension, ${ }^{24,25}$ congenital heart diseases $^{26}$ and arrhythmogenic RV dysplasia. ${ }^{27}$ The latest guidelines of American Society of 
Echocardiography and the European Association of Cardiovascular Imaging have recommended $\mathrm{RV}$ strain for clinical use as a sensitive and reproducible index of $\mathrm{RV}$ performance..$^{13,28}$ The current guideline recommends the normal value of RV strain $-29 \pm 4.5$, which was close to the value of our control group. However, RV strain of FMF patients was significantly lower. The reduction of the RV GLS in FMF patients is a novel finding to the best our knowledge. In the light of these findings, beyond the conventional RV assessment, novel RV function evaluation techniques such as Tei index and RV strain may provide useful information about RV clinical and subclinical functions, particularly in systemic diseases.

The most important limitation of our study was the relatively small number of patients. Moreover, when conducting an echocardiographic study, intra- and inter-observer variability is high. Adding cardiac magnetic resonance imaging would have added more precise data when comparing RV function assessment. Larger-size multicenter studies are needed to clarify the exact estimation of RV functions in FMF patients.

In conclusion, we have investigated the left and right ventricular systolic functions using STE in addition to conventional echocardiographic evaluation in adult FMF patients and demonstrated that RV Tei index and GLS were impaired in adults with FMF during the attack-free period.

\section{Acknowledgements}

Authors thank Dr. Ömer Yiğiner for his valuable contribution in the statistical analyses of the study.

\section{Declaration of conflicting interests}

The authors declared no conflicts of interest with respect to the authorship and/or publication of this article.

\section{Funding}

The authors received no financial support for the research and/or authorship of this article.

\section{REFERENCES}

1. Padeh S, Berkun Y. Familial Mediterranean fever. Curr Opin Rheumatol 2016;28:523-9.

2. Sohar E, Gafni J, Pras M, Heller H. Familial Mediterranean fever. A survey of 470 cases and review of the literature. Am J Med 1967;43:227-53.
3. La Regina M, Nucera G, Diaco M, Procopio A, Gasbarrini G, Notarnicola C, et al. Familial Mediterranean fever is no longer a rare disease in Italy. Eur J Hum Genet 2003;11:50-6.

4. Konstantopoulos K, Kanta A, Deltas C, Atamian V, Mavrogianni D, Tzioufas AG, et al. Familial Mediterranean fever associated pyrin mutations in Greece. Ann Rheum Dis 2003;62:479-81.

5. Kotone-Miyahara Y, Takaori-Kondo A, Fukunaga K, Goto M, Hayashino Y, Miki M, et al. E148Q/ M694I mutation in 3 Japanese patients with familial Mediterranean fever. Int J Hematol 2004;79:235-7.

6. Kees S, Langevitz P, Zemer D, Padeh S, Pras M, Livneh A. Attacks of pericarditis as a manifestation of familial Mediterranean fever (FMF). QJM 1997;90:643-7.

7. Ross R. The pathogenesis of atherosclerosis: a perspective for the 1990s. Nature 1993;362:801-9.

8. Akdogan A, Calguneri M, Yavuz B, Arslan EB, Kalyoncu U, Sahiner $\mathrm{L}$, et al. Are familial Mediterranean fever (FMF) patients at increased risk for atherosclerosis? Impaired endothelial function and increased intima media thickness are found in FMF. $J$ Am Coll Cardiol 2006;48:2351-3.

9. Baysal T, Peru H, Oran B, Sahin TK, Koksal Y, Karaaslan S. Left ventricular diastolic function evaluated with tissue Doppler imaging in children with familial Mediterranean fever. Clin Rheumatol 2009;28:23-8.

10. Ozdemir O, Agras PI, Aydin Y, Abaci A, Hizli S, Akkus HI, et al. Assessment of cardiac functions using tissue Doppler imaging in children with familial Mediterranean fever. Cardiol Young 2012;22:188-93.

11. Ceylan Ö, Özgür S, Örün UA, Doğan V, Yllmaz $\mathrm{O}$, Keskin $\mathrm{M}$, et al. Assessment of left ventricular functions with tissue Doppler, strain, and strain rate echocardiography in patients with familial Mediterranean fever. Anatol J Cardiol 2015;15:663-8.

12. Livneh A, Langevitz P, Zemer D, Zaks N, Kees $\mathrm{S}$, Lidar $\mathrm{T}$, et al. Criteria for the diagnosis of familial Mediterranean fever. Arthritis Rheum 1997;40:1879-85.

13. Lang RM, Badano LP, Mor-Avi V, Afilalo J, Armstrong A, Ernande L, et al. Recommendations for cardiac chamber quantification by echocardiography in adults: an update from the American Society of Echocardiography and the European Association of Cardiovascular Imaging. $\mathrm{J}$ Am Soc Echocardiogr 2015;28:1-39.e14.

14. Galiuto L. The Echo Manual. 3rd ed. Oxford: Oxford University Press; 2007.

15. Tei C, Ling LH, Hodge DO, Bailey KR, Oh JK, Rodeheffer RJ, et al. New index of combined systolic and diastolic myocardial performance: a simple and reproducible measure of cardiac function--a study in normals and dilated cardiomyopathy. $\mathrm{J}$ Cardiol 1995;26:357-66.

16. Voigt JU, Pedrizzetti G, Lysyansky P, Marwick TH, Houle H, Baumann R, et al. Definitions for a common 
standard for 2D speckle tracking echocardiography: consensus document of the EACVI/ASE/Industry Task Force to standardize deformation imaging. J Am Soc Echocardiogr 2015;28:183-93.

17. Tavil Y, Ureten K, Oztürk MA, Sen N, Kaya MG, Cemri $\mathrm{M}$, et al. The detailed assessment of left and right ventricular functions by tissue Doppler imaging in patients with familial Mediterranean fever. Clin Rheumatol 2008;27:189-94.

18. Caliskan M, Gullu H, Yilmaz S, Erdogan D, Unler GK, Ciftci O, et al. Impaired coronary microvascular function in familial Mediterranean fever. Atherosclerosis 2007;195:e161-7.

19. Tei C, Dujardin KS, Hodge DO, Bailey KR, McGoon $\mathrm{MD}$, Tajik AJ, et al. Doppler echocardiographic index for assessment of global right ventricular function. $\mathrm{J}$ Am Soc Echocardiogr 1996;9:838-47.

20. Sebbag I, Rudski LG, Therrien J, Hirsch A, Langleben D. Effect of chronic infusion of epoprostenol on echocardiographic right ventricular myocardial performance index and its relation to clinical outcome in patients with primary pulmonary hypertension. Am J Cardiol 2001;88:1060-3.

21. Bruch C, Schmermund A, Marin D, Katz M, Bartel $\mathrm{T}$, Schaar J, et al. Tei-index in patients with mildto-moderate congestive heart failure. Eur Heart $\mathrm{J}$ 2000;21:1888-95.

22. Yilmaz R, Gencer M, Ceylan E, Demirbag R. Impact of chronic obstructive pulmonary disease with pulmonary hypertension on both left ventricular systolic and diastolic performance. $\mathrm{J}$ Am Soc Echocardiogr 2005;18:873-81.
23. Kim WH, Otsuji Y, Yuasa T, Minagoe S, Seward JB, Tei C. Evaluation of right ventricular dysfunction in patients with cardiac amyloidosis using Tei index. J Am Soc Echocardiogr 2004;17:45-9.

24. Haeck ML, Scherptong RW, Marsan NA, Holman ER, Schalij MJ, Bax JJ, et al. Prognostic value of right ventricular longitudinal peak systolic strain in patients with pulmonary hypertension. Circ Cardiovasc Imaging 2012;5:628-36.

25. Fine NM, Chen L, Bastiansen PM, Frantz RP, Pellikka PA, Oh JK, et al. Outcome prediction by quantitative right ventricular function assessment in 575 subjects evaluated for pulmonary hypertension. Circ Cardiovasc Imaging 2013;6:711-21.

26. Alghamdi $\mathrm{MH}$, Mertens L, Lee W, Yoo SJ, Grosse-Wortmann L. Longitudinal right ventricular function is a better predictor of right ventricular contribution to exercise performance than global or outflow tract ejection fraction in tetralogy of Fallot: a combined echocardiography and magnetic resonance study. Eur Heart J Cardiovasc Imaging 2013;14:235-9.

27. Vitarelli A, Cortes Morichetti M, Capotosto L, De Cicco V, Ricci S, Caranci F, et al. Utility of strain echocardiography at rest and after stress testing in arrhythmogenic right ventricular dysplasia. Am J Cardiol 2013;111:1344-50.

28. Muraru D, Onciul S, Peluso D, Soriani N, Cucchini U, Aruta P, et al. Sex- and Method-Specific Reference Values for Right Ventricular Strain by 2-Dimensional Speckle-Tracking Echocardiography. Circ Cardiovasc Imaging 2016;9:e003866. 